\title{
Fractured J-Wire Stylet Presenting as an Intrauterine Foreign Body
}

\author{
GARY S. NAKHUDA, M.D., MELVIN T. THORNTON, M.D., \\ and MARK V. SAUER, M.D.
}

\begin{abstract}
A 32-year old gravida 0 was noted to have a persistent echo dense reflection on her transvaginal ultrasound after undergoing saline infused sonohysterography during IVF work-up. Diagnostic hysteroscopy discovered a 1-cm remnant of a J-wire embryo transfer catheter embedded in a false passage created just below the apparently normal uterine cavity. (J GYNECOL SURG 20:25)
\end{abstract}

\section{INTRODUCTION}

$\mathbf{T}$ He most ReCEnT report from the Centers for Disease Control and Prevention notes that nearly 100,000 cycles of assisted reproduction are performed in the United States annually. ${ }^{1}$ Most, if not all, of these patients underwent examinations of the uterine cavity prior to embryo transfer using either sonohysterography or hysterosalpingography. Although these procedures are relatively noninvasive, most reported complications refer to the rare pelvic infections that are known to occur following the infusion of saline or oil-based contrast media. ${ }^{2}$

Office-based saline infusion sonohysterography (SIS) is often preferred over HSG because it is relatively simple and expedient to perform, without the need for radiologic equipment or radiation exposure. Because the tubal status of the patient is not critical to image prior to in vitro fertilization (IVF), SIS is typically used to ensure that intrauterine pathology (i.e., synchiael adhesions, polyps, cervical stenosis) does not exist prior to undergoing a treatment cycle. The safety and sensitivity of this method for infertility patients has been well-described. ${ }^{3}$

We report an unusual complication in a patient following SIS, in which a J-wire catheter (Cook $\mathrm{Ob} / \mathrm{Gyn}$, Bloomington, IN) was used to introduce saline into the uterine cavity. J-wire catheters are commonly used in many fields of medicine including cardiology, endovascular surgery, critical care, and interventional radiology. The device consists of a wire guide with a flexible tip, providing the wire with "memory," such that difficult contours may be navigated more easily. Once the wire is in place, the outer sheath is advanced over the wire, the wire is removed, and the inner catheter is introduced through the outer sheath. Complications have been reported with the use of J-wires in other fields. ${ }^{4-6}$ This is the first report of a remnant tip left behind following its use for assisted reproduction.

Department of Obstetrics and Gynecology, Division of Reproductive Endocrinology, College of Physicians \& Surgeons, Columbia University, New York, NY. 


\section{CASE REPORT}

The patient was 32-year old gravida 0 who was enrolled in the assisted reproduction program at Columbia University College of Physicians \& Surgeons, New York, NY, for treatment of malefactor infertility. Prior to beginning her cycle, she had undergone a SIS, which reviewed a "polyp" within what appeared to be an otherwise normal endometrial cavity. The physician performing the procedure had also reported severe cervical stenosis, requiring the use of a J-wire catheter to infuse the saline after he failed to enter the cavity with a balloon-tipped catheter normally used to perform the SIS (Ackrad Laboratories, Cranford, NJ). The patient reported that she experienced a great deal of cramping during and immediately after the procedure. She also noted vaginal bleeding for 24 hours post-SIS but was told by a physician that this was not unusual.

On review of the SIS it appeared that the patient had a mid-fundal polyp and was referred for hysteroscopy and polypectomy. With the patient under conscious sedation, an outpatient hysteroscopy was undertaken to evaluate the cavity. An hysteroscope with a 3-mm outer sheath was introduced after minimal dilation. A foreign body, consistent with a fragment of metal wire, was visualized in the uterine cavity, apparently embedded into the endometrium (Fig. 1 shows the embedded J-wire). Hysteroscopic graspers introduced through the operative sheath were used to remove the object from the uterus. No polyp was seen.

It appeared that a false passage had been created in the lower uterine fundus at the point of anteflexion of the cervix, probably with the balloon-tipped SIS catheter. Hydrodissection may have enlarged the area into a pseudo-cavity. The J-wire tip was noted to be firmly embedded in the myometrium at the superior aspect of this cavity. It is likely that, in withdrawing the inner titanium tipped stylet through the flexed outer catheter, the tip fractured at a point about 1 $\mathrm{cm}$ from the end of the wire (Fig. 2 shows the breaking point of the J-wire) and was left behind unnoticed.

Upon reviewing the original SIS films postoperatively, it was noted that the pseudocavity was directly below the endometrial stripe. However, given the thin nature of the endometrium in the early follicular phase of the menstrual cycle, it was easily missed at the time of the original reading.

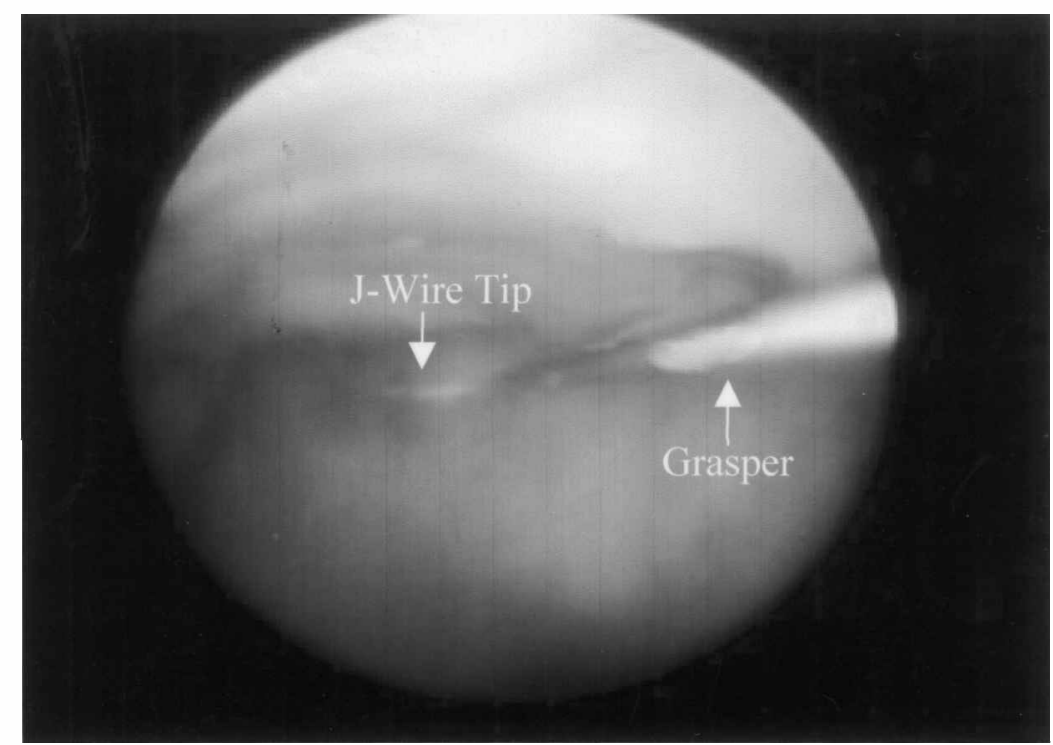

FIG. 1. The 1-cm tip of the J-wire stylet can be seen embedded in the upper fundus. 

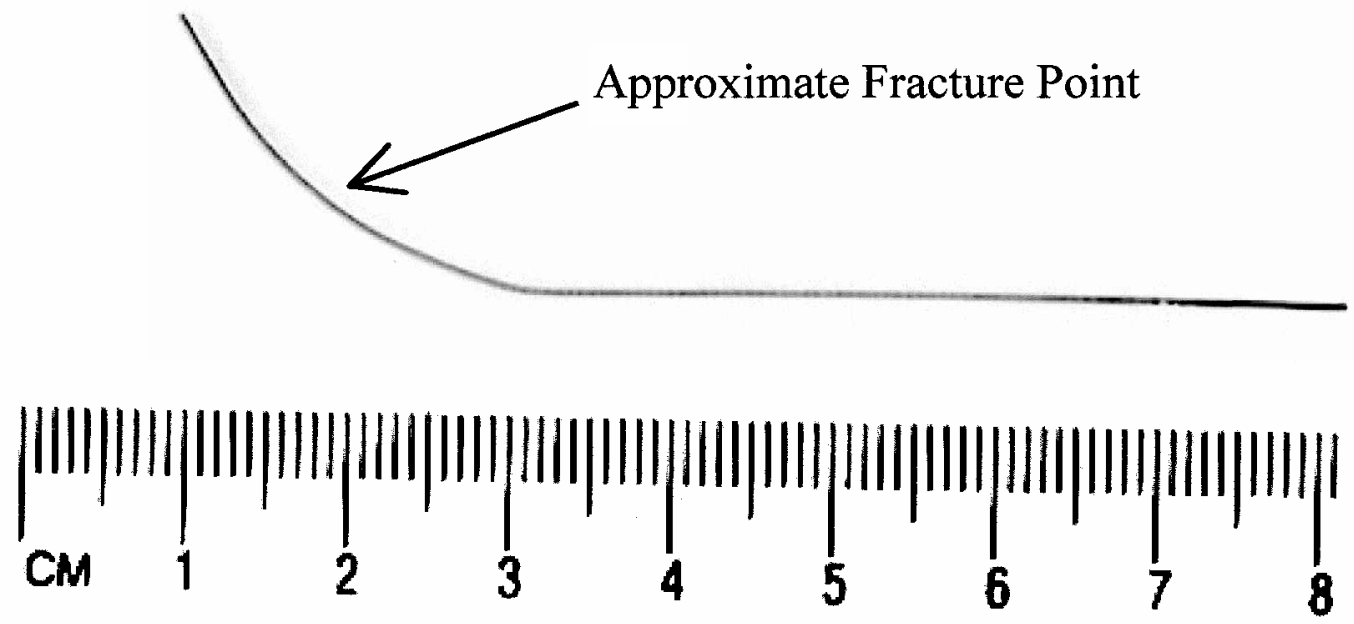

FIG. 2. Approximate breaking point of the J-wire.

\section{DISCUSSION}

This case illustrates the potential danger of using J-wire catheters in patients who are infertile. Although this is undoubtedly rare, the potential for fracturing the tip of the stylet and leaving a foreign body behind inside the uterus is possible. This complication has not been previously reported in patients undergoing infertility evaluation but complications of J-wire guides for vascular catheter placement have been reported repeatedly. Considering that J-wires are safely used on a routine basis, these complications are likely to be idiosyncratic and no suspicion of a manufacturer's defect has been reported for the use of J-wire catheter guides. Safeguards include the slow, deliberate, withdrawal of the inner stylet, visual inspection of the wire following its withdrawal, and avoidance of use in techniques for which a J-wire is not intended. In this particular case, none of these guidelines were apparently followed.

Office hysteroscopy was helpful for discovering the true nature of the abnormal SIS and correction of the problem. The discovery of the embedded device and its removal were important to the patient's well-being. Defining the anteflexed uterus and the normal endometrial cavity will assist in the ultimate placement of an embryo transfer catheter following her IVF procedure. Knowing that a false passage exists is important information for the physician performing the transfer. Using real-time ultrasound during the performance of the embryo transfer will ensure that the placement is indeed intrauterine and should improve the efficacy of the procedure.?

In summary, using J-wire devices to assist in performing intrauterine placement of catheters may be beneficial for navigating difficult cervical canals. Although technically similar to the placement of an embryo transfer catheter, SIS is not an intended use of the J-wire, and this procedure is probably performed better using a device specifically designed for it. Cases not amendable to SIS because of difficult anatomy may benefit from diagnostic hysteroscopy before beginning IVF treatment. Indeed, hysteroscopy has been suggested as a routine procedure for IVF evaluation and is associated with favorable outcome when used before controlled ovarian stimulation cycles. ${ }^{8}$ The potential for fracturing the J-wire tip does exist, and care must be taken to avoid this complication. Simple measures such as inspecting the device following its removal should reduce the likelihood of unintentially leaving a piece of the stylet in situ and, in the rare event of a fracture, allow for the piece's immediate removal. 


\section{REFERENCES}

1. Wright VC, Schieve LA, Reynolds MA, Jeng G. Assisted reproductivetechnology surveillance—United States, 2000. MMWR Surveill Summ 2003;52:1.

2. Bonnamy L, Marret H, Perrotin F et al. Sonohysterography: A prospective survey of results and complications in 81 patients. Eur J Obstet Gynecol Reprod Biol 2002;102:42.

3. Soares SR, Barbosa dos Reis MM, Camargos AF. Diagnostic accuracy of sonohysterography, transvaginal sonography, and hysterosalpingography in patients with uterine cavity diseases. Fertil Steril 2000;73:406.

4. Schou H. The double J-spring guide wire and its hazards: Partially retained double J-wire. A complication during central venous catheterization. Case report. Acta Anaesthesiol Scand 1988;32:158.

5. Panos AL, Common AA, Salerno TA. Unusual complication of J wire used for central venous cannulation. Ann Thorac Surg 1991;52:581.

6. Carpentier JP, Braz da Silva J, Choukroun G. Formation of a knot in a J spiral metallic guide: A complication of the Seldinger method. Cah Anesthesiol 1991;39:277.

7. Matorras R, Urquijo E, Mendoza R, et al. Ultrasound-guidedembryo transfer improves pregnancy rates and increases the frequency of easy transfers. Hum Reprod 2002;17:1762.

8. Mooney SB, Milki AA, Effect of hysteroscopy performed in the cycle preceding controlled ovarian hyperstimulation on the outcome of in vitro fertilization. Fertil Steril 2003;79:637.

Address reprint requests to:

Mark V. Sauer, M.D.

Department of Obstetrics and Gynecology

Columbia Presbyterian Medical Center

622 West 168th Street, PH16-28

New York, NY 10032

E-mail:mvs9@columbia.edu 